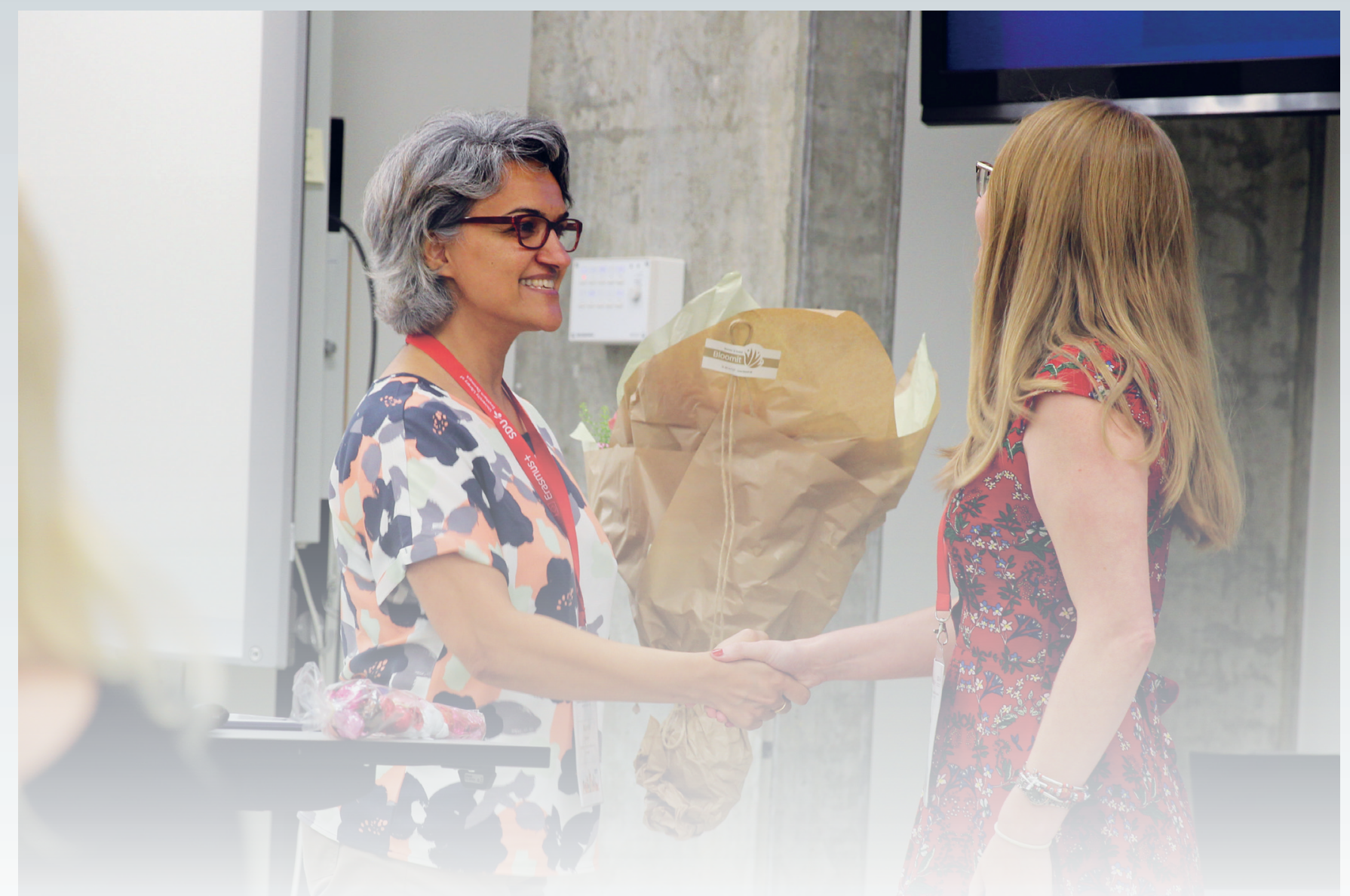

\title{
International Library Staff Week at SDUB
}

The first International Staff Week for Librarians (Erasmus+Program), was held at the University Library of Southern Denmark (SDUB), Odense, from 11 - 14 June 2019. The event was hosted by the library under Internationalization Project and the aim was to exchange of ideas, knowledge and best practices.

The event has been started planning in second half of 2018 and announced through IMOTION (Erasmus Staff Training webpage), LIBER, networking channels and social medias. We have received over 127 applications from 42 countries around the world and SDUB Internationalization committee has chosen 20 candidates from 17 countries with considering some highlighted criteria such as position level, experience and educational background.
The event was a platform for exchange, networking, sharing knowledge and best practices within and across professional areas at research libraries.

Discussions centred on the practical topics towards university libraries, with the various sessions featuring presentations, workshops, round table discussions, networking activities and cultural events.

The main themes of the week included, but not limited to, the following development areas/fields classified into:

- Information Literacy \& Teaching

- E-Learning

- Citizen Science and role of the libraries

- Research Support Services
Also, participants had opportunity to contribute to the program with a short presentation of their home institutions and sharing experiences.

Alongside the main sessions, a presentation on Danish Life and Workplace culture enriched the programme. Participants also had the chance to learn some Danish words at first day during "Meet \& Greet".

They also had the option to take part in the City tour and a cultural visit, also been invited to the evening networking event at a traditional restaurant in town.

The event documents (Welcome Package) distributed to delegates included the programme, brochure, practical information. 
Written by: Najmeh Shaghaei

Head of Library (Campus Sønderborg) \& Internationalisation Coordinator -SDUB,nas@bib.sdu.dk

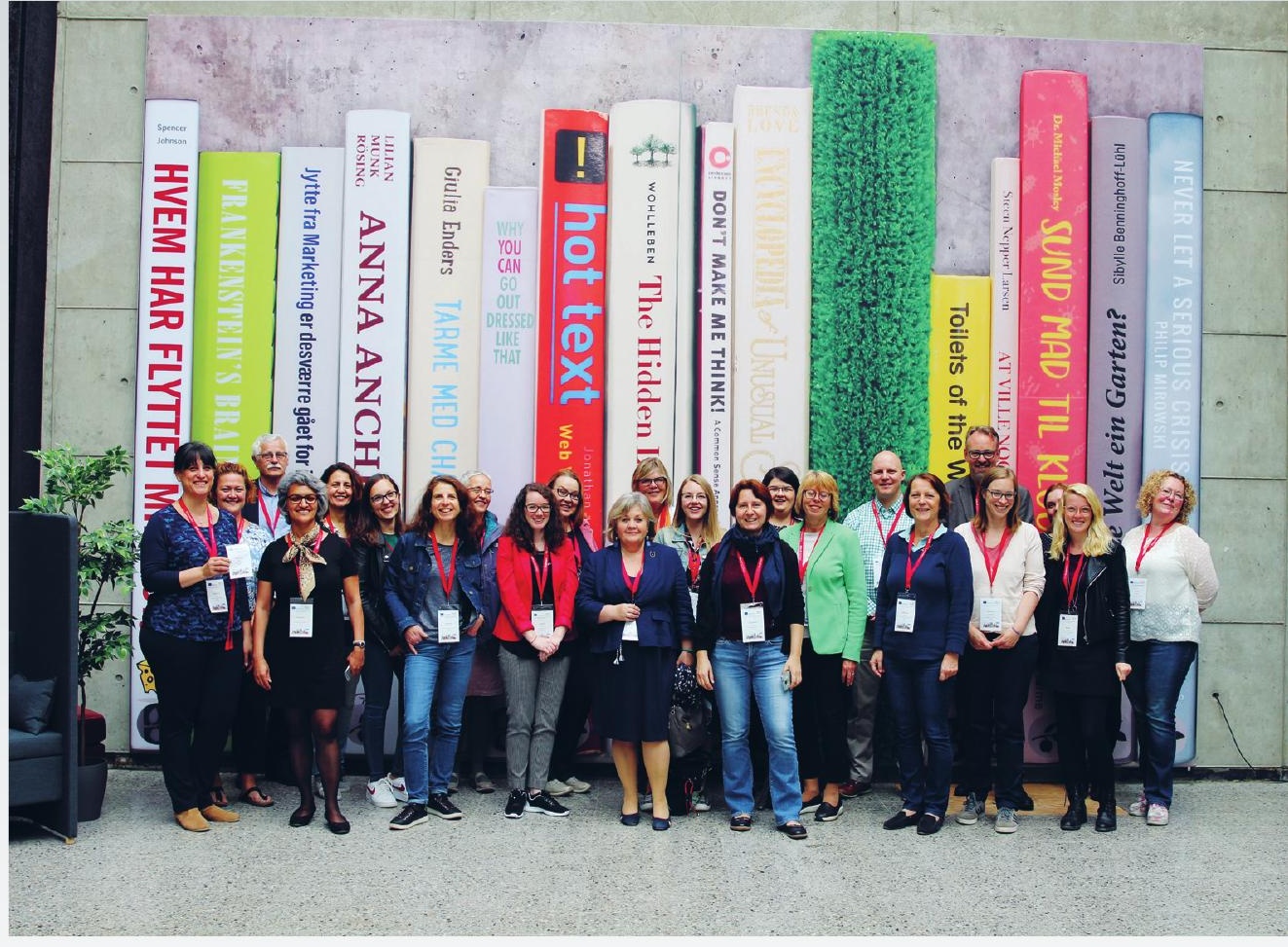

There was specific topic for each day during the week, and library colleagues had chance to present unique topics from their respective departments.

The first day started with Welcoming and Opening session at the library. The following representatives co-organizing and hosting university and library and supporting organizations addressed to participants with short welcoming speeches.

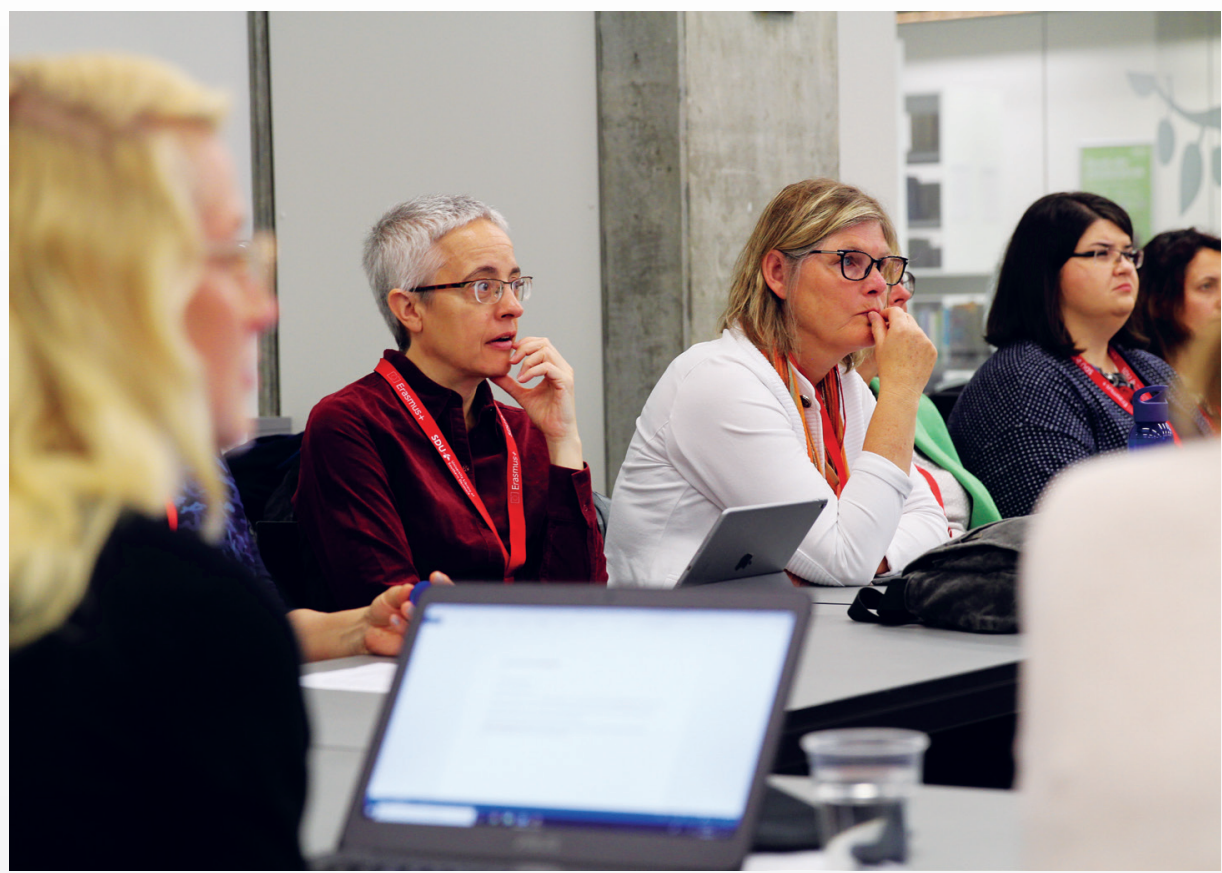

After welcome speeches, program continued by project group with "Meet \& Greet” session. Participants got to know each other through presenting themselves by adding their photos on the global map that showed where they come from. They also expressed their expectation from the week at SDUB.

The day continued by having networking lunch at the university restaurant and presenting Danish life and workplace culture by Annika Jarl from HR the day by the library and university tour.

Second day with theme of "Teaching and Learning Day" was held by different presentations and workshops by Jens Dam and his colleagues from Information section at the library. The topics included Information Literacy and Teaching, UX LIBS and E-Learning. In the afternoon, selected participants from the University of Worcester (UK), University of Zilina (Slovakia), Kaunas University of Technology (Lithuania) and Hanze University of Applied Sciences (Netherlands) had chance to present their libraries and highlighted services.

The third day with Theme of "Citizen Science Day" was held by presenting the interesting topic of Citizen Science department afterwards and finished 


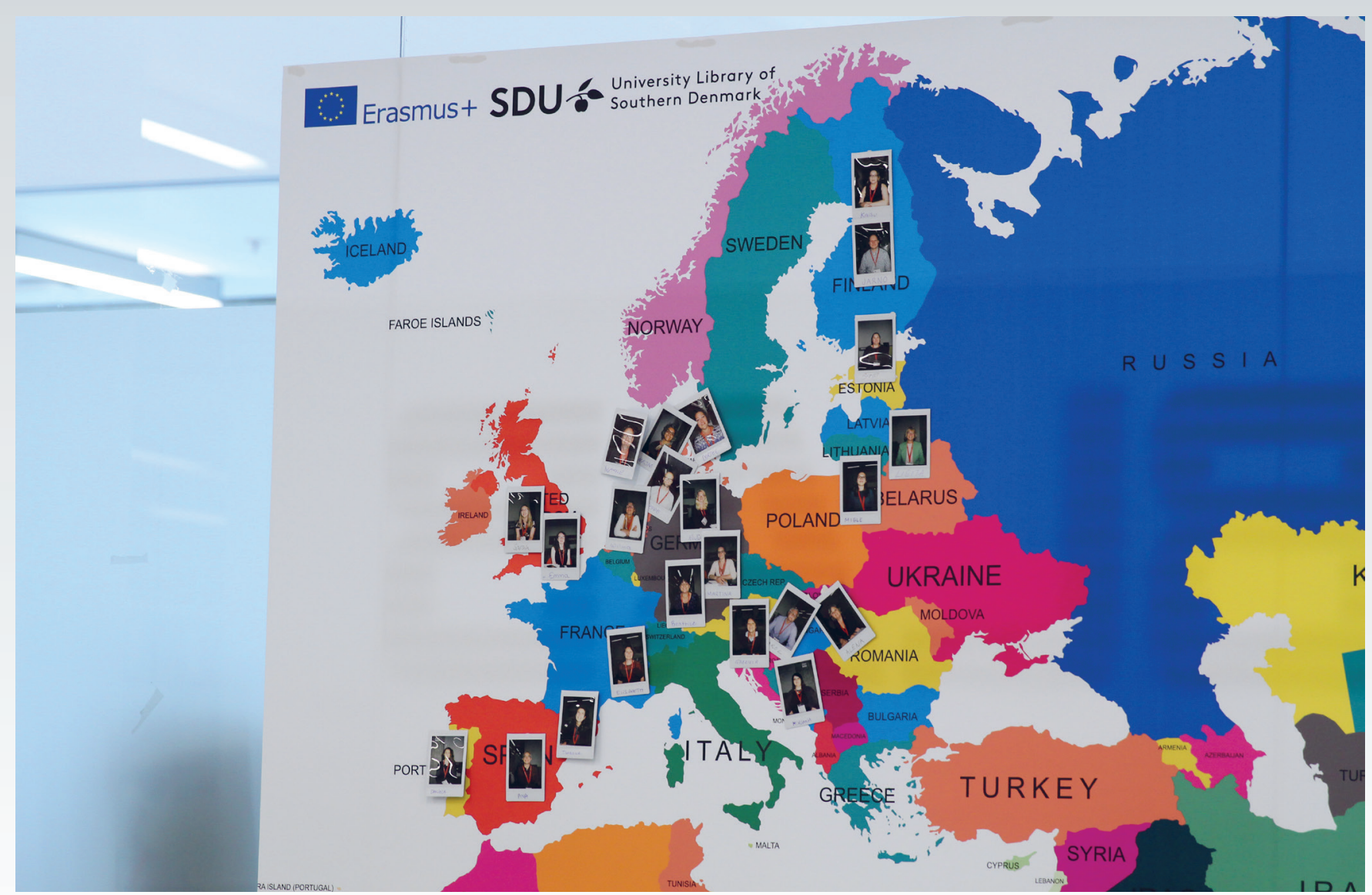

from different aspects. The day managed by Thomas Kaarsted, Anne Kathrine Overgaard and Prof. Jens Troelsen from SUND about Citizen Science and the role of libraries and the perspective of Faculty.

An afternoon free was organized for participants to explore the city, visiting H. C. Andersens House and enjoying an evening networking in a nice and cosy restaurant in town.

The last day of the week was organized with theme of "Research Day" and Bertil presented "Responsible Conduct of Research", Mogens and Jakob from Research service department presented "Special Collection" and organized our impressive Special Collection exhibition, also Anne Thorst Melbye presented PURE and SDU repository. The day finished with "Wrap-Up and Feedback" session. The success of the event was measured and inferred by the collected feedback, and a considerable amount of advice was collected from participants.
The first International Library Staff Week has become the most widescale, extensive, spectacular event hosted by SDUB as the first Erasmus +Program for Librarians in Denmark.

It is fair to conclude that the event was a great success! So many people have contributed in so many ways to turn this event into a smoothly running meeting with many very interesting presentations and a very good atmosphere for discussion and networking.

I owe much gratitude to SDUB management, LF, SDU International Director and HR department for their enormous and high-quality support, our excellent colleagues for their great effort and effective contribution, and project team for giving enormous amounts of time and energy for everything to fall into place as it has.

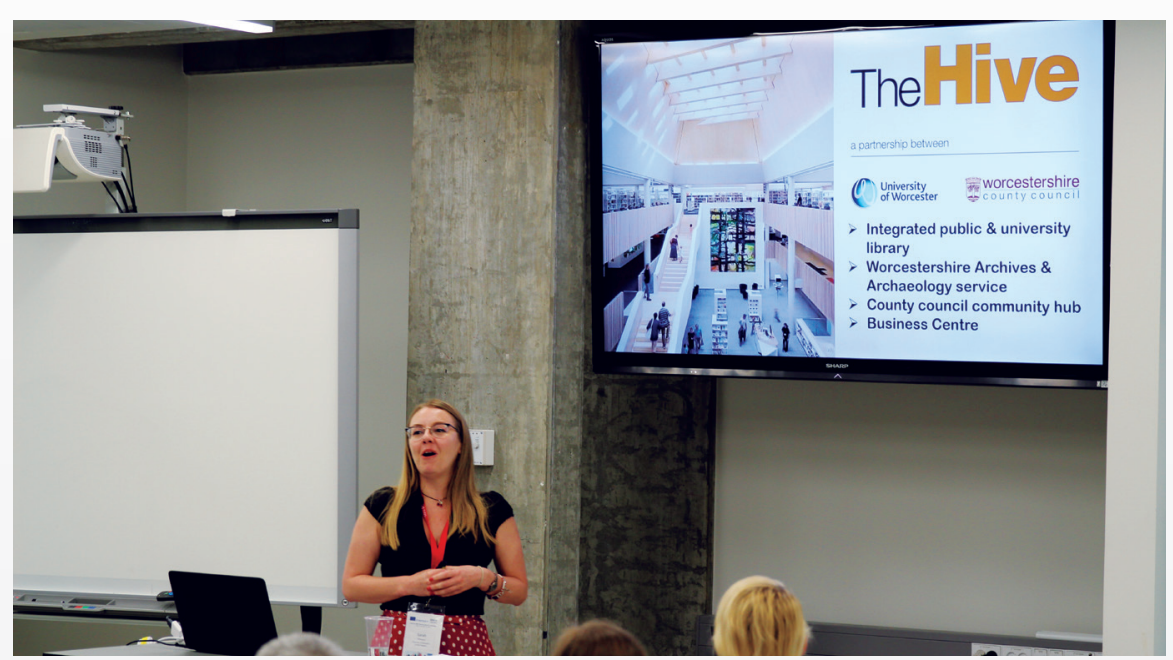

